\title{
Scales of the magnetic fields in the quiet Sun
}

\author{
A. López Ariste ${ }^{1}$ and A. Sainz Dalda ${ }^{2}$ \\ 1 THEMIS - CNRS UPS 853, C/ Vía Láctea s/n, 38200 La Laguna, Spain \\ e-mail: Arturo.Lopez@themis.iac.es \\ 2 Stanford-Lockheed Institute for Space Research, HEPL, Stanford University, Cypress Hall, 466 Via Ortega, Stanford, CA 94305, \\ USA
}

Received 30 September 2011 / Accepted 6 February 2012

\begin{abstract}
Context. The presence of a turbulent magnetic field in the quiet Sun has been unveiled observationally using different techniques. The magnetic field is quasi-isotropic and has field strengths weaker than $100 \mathrm{G}$. It is pervasive and may host a local dynamo.

Aims. We aim to determine the length scale of the turbulent magnetic field in the quiet Sun.

Methods. The Stokes V area asymmetry is sensitive to minute variations in the magnetic topology along the line of sight. Using data provided by Hinode-SOT/SP instrument, we performed a statistical study of this quantity. We classified the different magnetic regimes and infer properties of the turbulent magnetic regime. In particular we measured the correlation length associated to these fields for the first time.

Results. The histograms of Stokes V area asymmetries reveal three different regimes: one organized, quasi-vertical and strong field (flux tubes or other structures of the like); a strongly asymmetric group of profiles found around field concentrations; and a turbulent isotropic field. For the last, we confirm its isotropy and measure correlation lengths from hundreds of kilometers down to $10 \mathrm{~km}$, at which point we lost sensitivity. A crude attempt to measure the power spectra of these turbulent fields is made.

Conclusions. In addition to confirming the existence of a turbulent field in the quiet Sun, we give further prove of its isotropy. We also measure correlation lengths down to $10 \mathrm{~km}$. The combined results show magnetic fields with a large span of length scales, as expected from a turbulent cascade.
\end{abstract}

Key words. Sun: photosphere - techniques: polarimetric - Sun: magnetic topology - techniques: spectroscopic

\section{Introduction}

This work is dedicated to explore the properties of the turbulent magnetic field in the quiet Sun through the analysis of the asymmetries in the Stokes V profiles observed by Hinode-SOT/SP (Kosugi et al. 2007; Tsuneta et al. 2008) in a Zeeman-sensitive line. The existence of a magnetic field turbulent in nature in those places with weak Zeeman signals and absence of temporal coherence in the plasma flows is taken for granted and, from Sect. 2 and thereafter, we shall not discuss whether these fields are turbulent or not. Although our analysis provides further evidence of the existence of this turbulent field, we will assume that this existence is proven and make our analysis in that framework, studying the coexistence of those turbulent fields with others more structured in nature, whose existence is also beyond questioning. Despite that, but also because of that, we now dedicate a few lines to justify and put into context the turbulent nature of the magnetic field in most of the quiet Sun.

The existence of a turbulent magnetic field accompanying the turbulent plasma in the quiet Sun is not a theoretical surprise, rather the opposite. Upon the discovery of flux tubes in the photospheric network, Parker (1982) expressed surprise at the existence of these coherent magnetic structures and referred to them as extraordinary state of the field. He argued in that paper that only under conditions of temporal coherence of the plasma flows in the photosphere could these structures be stable. Consequently, one could expect to find them in the photospheric network where advection flows concur before dipping into the solar interior. Similar conditions can be found here and there in the internetwork in those intergranular lanes where plumes have grown strong enough to survive granular lifetimes. Everywhere else the high Reynolds number of the photospheric plasma does not allow any structure of any kind and a turbulent field, if anything, is to be expected. Other theoretical analyses (Petrovay 2001) confirm and insist on these turbulent fields. The first attempts of numerical simulations of magnetoconvection (Nordlund et al. 1992) revealed a magnetic field, whose field lines, away from downflows, are twisted and folded even for the low Reynolds numbers (kinetic and magnetic) and for the wrong ratio of both adimensional quantities. Independent of the existence of a local dynamo on which most of these simulations focus, the turbulent field is there.

Observationally, the picture has been different until recently. The discovery of flux tubes in the network (Stenflo 1973) together with the common observation of G-band bright points in high-resolution images of the photosphere has spread the idea that flux tubes are everywhere. Because inversion techniques for the measurement of the magnetic field mostly used Milne-Eddington atmospheric models that assume a single value of the magnetic field per line of sight, whenever they have been used in the quiet Sun, a single field value was attached to a point in the photosphere, which additionally spread the impression that it was the field of the flux tube. Doubts were cast on this observational picture of the quiet Sun when magnetic measurements using infrared lines produced fields for the same points different than measurements with visible lines. The wavelength dependence of the Zeeman effect sufficed to expose the fact that a single field could not be attached to a given point in the quiet 
Sun. Both measurements were right, in the sense at least that they were measuring different aspects of the complex magnetic topology of the quiet Sun. Turbulent fields had in the meantime been the solution offered by those observing the quiet Sun through the Hanle effect. The absence of Stokes U in those measurements and the high degree of depolarization of the lines pointed to a turbulent field as the only scenario fitting their observations. Unfortunately, the difficulties both in the observations (very low spatial and temporal resolutions) and in the diagnostic (many subtle quantum effects involved) made the comparison with the observations using Zeeman effect difficult. The advent of the statistical analysis of Zeeman observations has solved the problem. First it was the observation that the quiet Sun, if one excludes the network and strong magnetic patches from the data, looks suspiciously similar independent of the position on the solar disk that one is observing (Martínez González et al. 2008b). This independence of the measurements with the viewing angle pointed toward isotropy, a characteristic of the quiet Sun fields later confirmed by Asensio Ramos (2009). Then came the realization that the average longitudinal flux density measured in the quiet Sun at different spatial resolutions was roughly the same (Lites 2002; Martínez González et al. 2010). This could only be interpreted that either the field was already resolved, but obviously it was not, or that the observed signals were just the result of a random addition of many magnetic elements. Within the limit of large numbers the amplitude of this fluctuation only depends on the square root of the size and not linearly, as expected for a nonresolved flux tube. The turbulent field is in this way unveiled by the statistical analysis of Zeeman effect, and it was shown that Zeeman signatures in the quiet Sun were often merely statistical fluctuations of the turbulent field and not measurements of the field itself (López Ariste et al. 2007).

The solar magnetic turbulence was therefore explored in a statistical manner. Furthermore, it was explored assuming that different realizations of the magnetic probability distribution functions sit side by side. In this approximation one can compute the resulting polarization by just adding up the individual contributions of each magnetic field. The Stokes V profile caused by the Zeeman effect of each individual magnetic field will be antisymmetric with respect to the central wavelength, with one positive and one negative lobe. The areas of the two lobes of every profile are identical and their addition, the area asymmetry, will be zero. Adding many such polarization profiles will alter the resulting profile, but the area asymmetry of the final profile will always be zero. A completely different result is obtained if we consider the different realizations of the magnetic field probability distribution function placed one after the other along the line of sight. Computing the resulting polarization profile now requires integrating the radiative transfer equation for polarized light in a non-constant atmosphere. If the variations in the magnetic field along the line of sight are associated with velocities, the integration results in a profile that lacks any particular symmetry.

Therefore, measuring and analyzing the area asymmetry of the Stokes V profiles in the quiet Sun provides information on the properties of the turbulent magnetic field along the line of sight, in contrast with the previous studies, which only explored this turbulence in terms of accumulation of magnetic elements in a plane perpendicular to the line of sight. At disk center, the line of sight means exploring those fields with depth, while near the limb it means exploring fields sitting side by side. Comparing asymmetries in statistical terms from quiet regions at different heliocentric angles provides us with a probe on the angular dependence of the magnetic fields, and this is one of the purposes of this paper. In Sect. 2 we describe the asymmetries observed by
Hinode-SOT in these terms and recover the three expected magnetic regimes: the structured and mostly vertical strong fields (strong in terms of quiet Sun magnetism), the turbulent, ubiquitous, disorganized and weak fields and a class of profiles with strong asymmetries that can be observed at those places where the line of sight crosses from one regime to the other, from turbulent to organized.

Focusing on these profiles assigned to turbulent magnetic fields, the value of the area asymmetry can be linked with the dominant scales of variation of the magnetic field. The results on stochastic radiative transfer that allow us to make that link are recalled in Sect. 3, in particular those of Carroll \& Kopf (2007). Thanks to those works we can quantitatively determine the correlation length of the magnetic field for every value of area asymmetry. From this determination we attempt to give an energy spectrum for the magnetic turbulence at scales below the spatial resolution. For this attempt, we will use the longitudinal flux density as a lower boundary to the field strength, and hence to the magnetic energy, and plot it versus the correlation length already determined. The approximations and simplifications made to reach this result may appear excessive to the reader. We argue that it is important not as an energy spectrum to be compared to numerical simulations or to theoretical considerations, but as a first attempt that follows what we consider to be a promising tool and method for more elaborated and reliable determinations of the magnetic energy spectrum. We also stress that through the proxy of the asymmetries of the profiles (seen through the models, tools, and results of stochastic radiative transfer), we can access scales of variation of the magnetic field 10 times smaller than the diffraction limit of our best instruments and probably smaller or comparable to the mean free path of the photons in the photosphere.

\section{Statistics of area asymmetries of the Stokes V profile}

To collect data on asymmetries of the Stokes V profiles in the quiet Sun at different heliocentric angles, we examined data from the SOT/SP instrument on board Hinode. Several large area scans of the quiet Sun at different positions on the solar disk were selected. Table 1 summarizes the observational features of those data. The spatial sampling $\left(0.15^{\prime \prime} \times 0.16^{\prime \prime}\right)$ and spectral sampling (roughly $21 \mathrm{m \AA}$ ) were the same for all observing runs, but the exposure times changed from one to other. The data were calibrated using the standard procedure, which is available in SolarSoft and has been developed by Lites. The area asymmetry is measured as the integral of the profile over the Fe I $6302.5 \AA$ line, normalized to the total enclosed area, and with corrected polarity. Before this measurement a denoising based on PCA (Principal Component Analysis) of the data has been performed (Martínez González et al. 2008a). This allows one to establish true noise and signal levels for the signal. In brief, the eigenvectors of the correlation matrix of each data set are computed at well-defined heliocentric angles. The data are reconstructed with only the ten first eigenprofiles. The rest are added to provide a measurement of noise. Histograms of this residual show the unmistakable Gaussian distribution shape of noise with typical values of $5 \times 10^{-4}$ the intensity of the continuum for the maximum of the distribution.

The first eigenprofile is, as usual in PCA techniques, the average eigenprofile and accordingly presents a nice antisymmetric shape with two well-defined lobes. This eigenprofile is used to automatically detect the polarity of every observed profile: a 
Table 1. Observational parameters of the data set.

\begin{tabular}{lcccccc}
\hline \hline Date time & $\begin{array}{c}X \text { scale } \\
\left({ }^{\prime \prime}\right)\end{array}$ & $\begin{array}{c}Y \text { scale } \\
\left({ }^{\prime \prime}\right)\end{array}$ & $\begin{array}{c}(X, Y) \\
\left({ }^{\prime \prime},{ }^{\prime \prime}\right)\end{array}$ & $\mu$ & $\begin{array}{c}\text { Exp. time } \\
(\mathrm{s})\end{array}$ & $S / N$ \\
\hline $2007-09-01 ~ 20: 35$ & 0.15 & 0.16 & $(-153.1,922.9)$ & 0.224 & 8.0 & 549. \\
2007-09-06 15:55 & 0.15 & 0.16 & $(-34.6,7.0)$ & 0.999 & 8.0 & 1150. \\
2007-09-09 07:05 & 0.15 & 0.16 & $(646.8,7.2)$ & 0.739 & 9.6 & 957. \\
$2007-09-2701: 01$ & 0.15 & 0.16 & $(-1004 ., 7.5)$ & 0.000 & 12.8 & 887. \\
\hline
\end{tabular}

Notes. The spectral sampling was $21 \mathrm{~m} \AA$ for all observation runs.

positive coefficient for this eigenprofile indicates a positive polarity profile. With this simple test we can automatically assign an unambiguous sign for the area asymmetry for all but a few anomalous cases, which can be easily disregarded because of their scarcity. The resulting sign of the area asymmetry is therefore related to the area of the blue lobe of Stokes V compared to that of the red lobe, and has no relationship with the polarity of the field. A positive area asymmetry results when the red lobe encloses a larger area than the blue lobe.

Using a scalar parameter like the area asymmetry to describe the many different spectral features that a Stokes V profile can present can be perceived as simplistic considering the magnetically and thermodynamically complex atmospheres in which it is formed. Studies and classifications of those shapes have been made and interpreted in the past (Sánchez Almeida \& Lites 1992; Viticchié et al. 2011) and we refer to them for details on this aspect of asymmetries. No doubt, a scalar like the area asymmetry hides all that richness and we should worry about the possibility that our conclusions could be polluted or invalidated because of that reason. In face of that criticism we claim that the area asymmetry, contrary to the amplitude asymmetry, allows a relatively easy analytical computation under unspecified variations of the atmospheric parameters (López Ariste 2002). The shape of the profiles is implicitly taken into account in these computations and, however complex, it does not alter the known dependencies of the area asymmetry on magnetic and velocity fields. Nevertheless, the shape of the profile, or of any particular spectral feature, can be attributed to particular variations in the atmosphere, something that cannot be achieved with only a value for the area asymmetry. As an example, we mention below the interpretation of single-lobe $\mathrm{V}$ profiles as produced in atmospheres with jumps along the line of sight. Because this work is limited to the area asymmetry, we avoid any conclusion on particular conditions along the line of sight. We will therefore describe the atmospheres through a simple correlation length with no additional details on the geometry of the fields, details that the analysis of profile shapes may eventually provide.

Figure 1 shows the data on area asymmetry for four different heliocentric angles $(\mu=1,0.88,0.7$, and 0.2$)$. The left column shows the typical histogram of frequency as a function of signed area asymmetry. The position of the maxima of these histograms is given as a vertical dashed line. The histograms are quite common bell-shaped distributions with a slight bias toward negative area asymmetries, that is, toward profiles with a blue lobe larger than the red one. That bias is stronger as we approach the solar limb (Martínez Pillet et al. 1997). Larger blue lobes as observed have been observed in the past in active regions and network fields (Solanki \& Stenflo 1984). They have been traditionally interpreted as being the result of the more usual gradients in the solar atmosphere (Solanki \& Pahlke 1988). More precise information can be obtained if we make a $2 \mathrm{D}$ histogram as a function of the signal amplitude. This signal amplitude of the Stokes V profile in quiet Sun conditions can be safely interpreted as longitudinal flux density. Although given in terms of polarization levels, it can as a rule of thumb be interpreted as $\times 1000 \mathrm{MW} / \mathrm{cm}^{2}$. These histograms are shown in the right column of Fig. 1. In them we plot in gray levels (color online) the histogram of area asymmetry of profiles with that signal amplitude. Indeed, to better display the strong, organized but relatively rare fields, the histograms are plotted not linearly but logarithmically. What is apparent in these plots is, first, a component of strong but weakly asymmetric fields with amplitudes higher than roughly $3 \%$ ( or $30 \mathrm{MW} / \mathrm{cm}^{2}$ ). This class of fields is an important contribution to the histogram at and near disk center but is only marginally important near the limb. The obvious interpretation is that these fields are the structured non-turbulent and mostly vertical fields that can be found in the photospheric network or in particular intergranular lanes. This hypothesis can be confirmed by inspecting the actual magnetograms compared with the intensity maps (not shown here), rather than in the histograms as presented. Because they are mostly vertical, it is clear that their signature in Stokes $\mathrm{V}$ will diminish as we approach the limb where these fields are seen transversally. Their asymmetries are small and centered around zero in all data sets. This indicates that these are very coherent structures with few variations (magnetic and velocity fields alike) along the line of sight.

Except for this class of strong and weakly asymmetric profiles, the histograms are dominated by fields with low amplitude values (though well above the noise limit) that can produce almost any possible asymmetry from 0 to \pm 1 . The most extreme asymmetries correspond to profiles with just one lobe, and we discuss them below. Now we concentrate on the distinction between this class of profiles with any asymmetry but weak amplitude and the symmetric and strong-amplitude class described in the previous paragraph. The separation between those two classes can better be seen with the help of Fig. 2, particularly the right column of $2 \mathrm{D}$ histograms. We computed the position of the maximum of the histogram for each value of the amplitude signal and plotted it versus amplitude in the left column of that figure. The strong fields have maxima at zero asymmetry. Maxima start drifting toward negative values for amplitudes below $5 \%\left(\right.$ or $50 \mathrm{MW} / \mathrm{cm}^{2}$ ) and we can place a boundary between the two classes at amplitudes of $3 \%$ (or $30 \mathrm{MW} / \mathrm{cm}^{2}$ ). We observe that the fields responsible for this change in asymmetry appear to be mostly insensitive to the heliocentric angle. This can be seen in Fig. 3 where the histograms were made exclusively with profiles with amplitudes below the boundary of $3 \%$ (or $30 \mathrm{MW} / \mathrm{cm}^{2}$ ). We overplotted in the same figure and at the same scale the histograms for all heliocentric angles to facilitate the comparison. The few differences with heliocentric angle that were seen in Fig. 1 have now almost disappeared. Only a small variability in the slope toward negative area asymmetries is noticeable now. This variability is in contrast with the almost perfect superposition of the histograms in their slope toward positive area asymmetries. We are unable to offer a definitive explanation for the variability in one of the slopes, but we nevertheless 

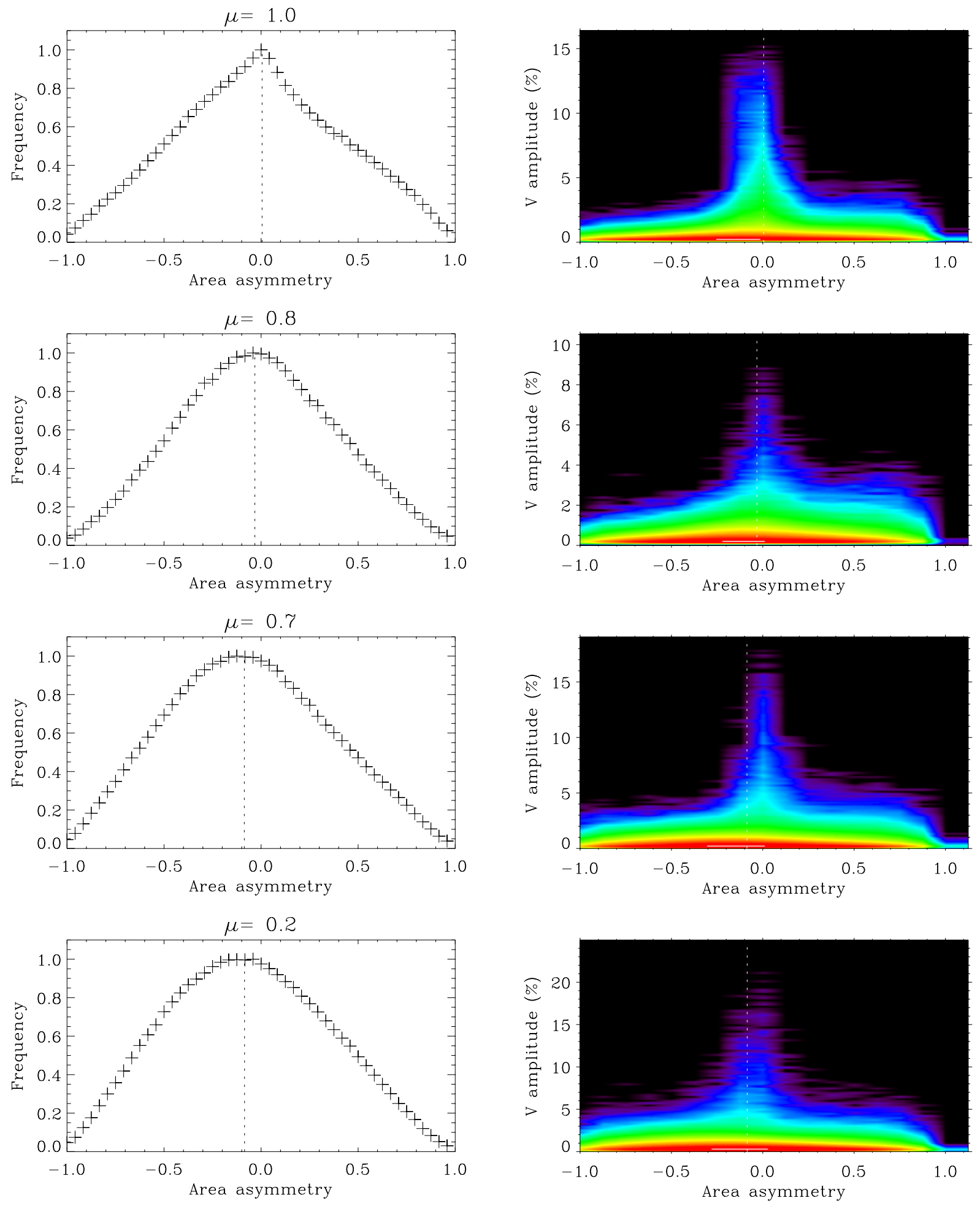

Fig. 1. Histograms for four different heliocentric angles (from top to bottom) of the observed asymmetry in Stokes V profiles of the Fe I line at $6302.5 \AA$. The left column presents the total histogram, while in the right column it is presented as a function of the amplitude of the profile in the ordinate axis and with histogram values in color. For better visibility the logarithm of the actual value is shown. A vertical dashed line marks the position of the maximum of the histogram in all plots.

stress the weak dependence of those histograms on the heliocentric angle. It is not proof, but yet suggestive evidence of the general isotropy of those fields that their signature in terms of area asymmetries is independent of the view angle under which they are observed.
It would be premature to identify any and all fields with amplitudes below $3 \%$ with the turbulent fields that pervade the quiet Sun. The histograms of Fig. 3 suggest the conclusion that they appear to be isotropic, which is an important characteristic of turbulent magnetic fields in the sense of fields frozen to 
A. López Ariste and A. Sainz Dalda: Scales of the magnetic fields in the quiet Sun
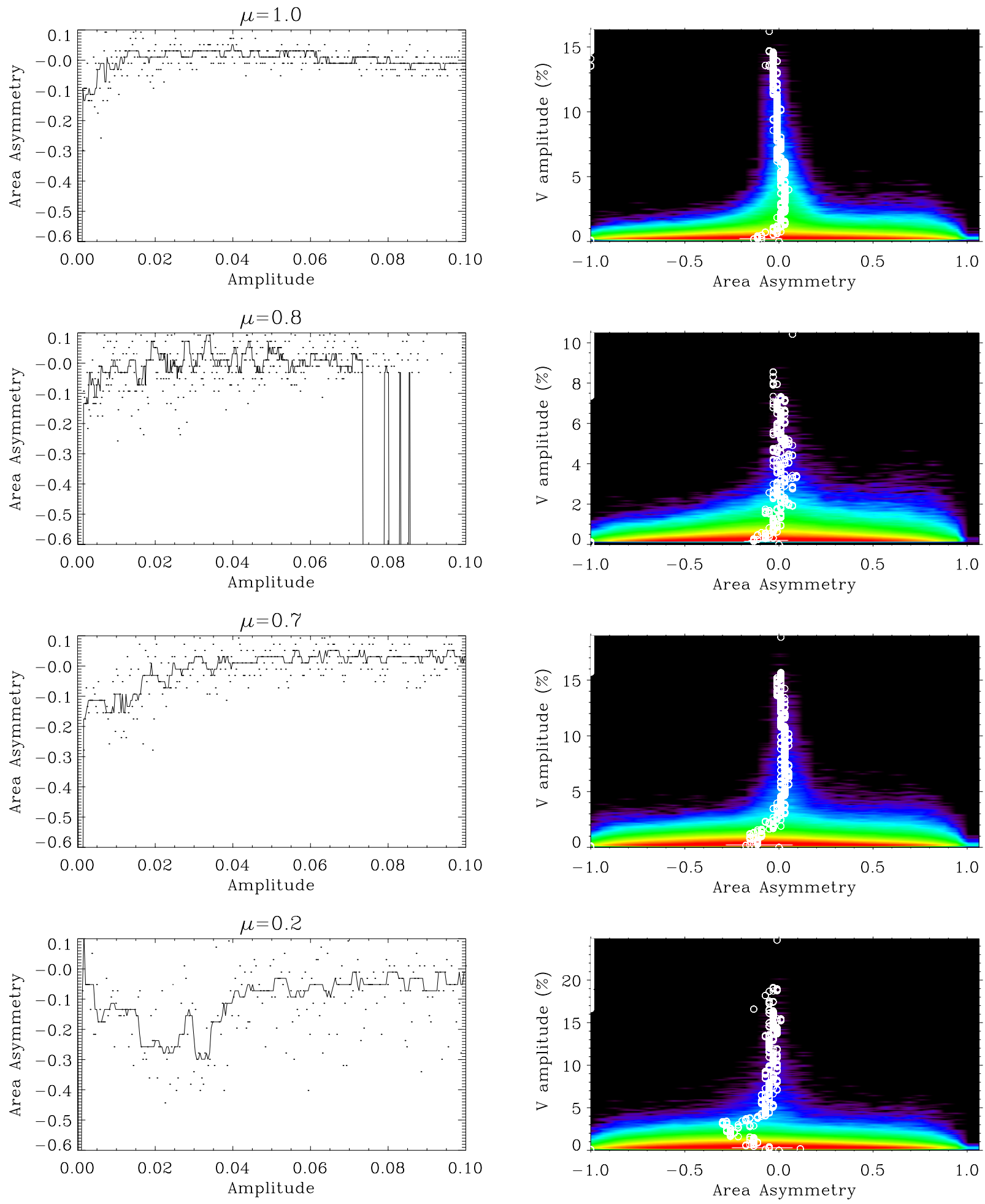

Fig. 2. Average value of the area asymmetry of Stokes $\mathrm{V}$ for different heliocentric angles and as a function of the amplitude of the signal. In the left column we present the actual measurements with a smoothed median line overplotted. In the right column this median line has been drawn over the 2D histograms of Fig. 1.

a turbulent photospheric plasma at high values of the plasma $\beta$ parameter. The reason for this caution is that radiative transfer of polarized light through an atmosphere at the microturbulent limit concludes that asymmetries larger on average than 0.53 are impossible (Carroll \& Kopf 2007). Therefore, those observed profiles with amplitudes below $3 \%$ but with extreme asymmetries cannot be considered as being formed by radiative transfer through turbulent fields. At this point, we recall that those anomalous single-lobed profiles, with extreme asymmetries, are not observed at random over the solar surface but are instead systematically observed over the boundaries of regions with concentrations of strong magnetic fields (Sainz Dalda et al. 2012). This realization suggests that the origin of those profiles is a line of sight that crosses from a weak disordered magnetic region to a strong and organized magnetic structure, with magnetic and velocity fields completely uncorrelated in one and the other 


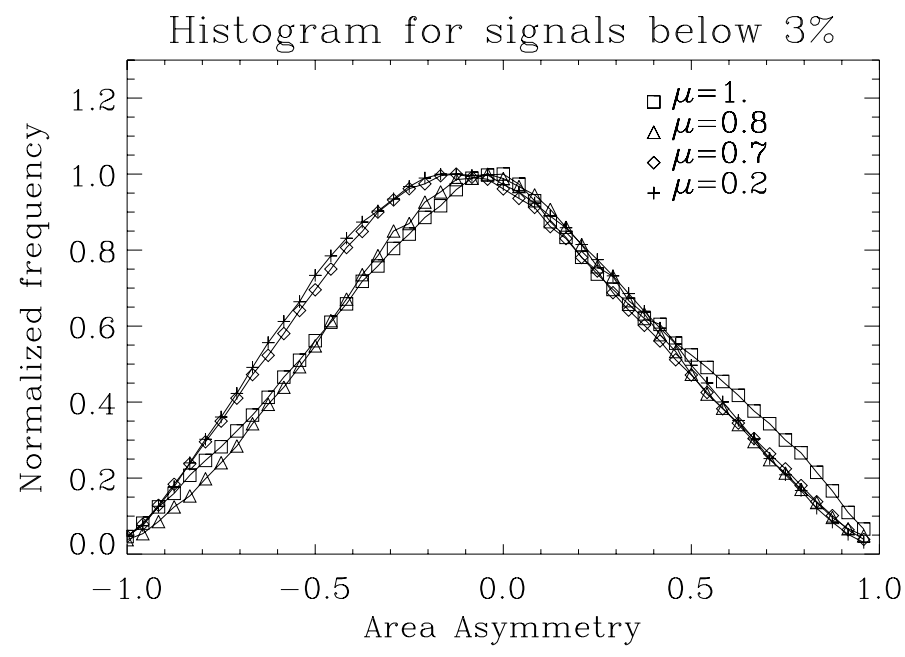

Fig. 3. Histograms of the asymmetries of the Stokes V profiles with amplitude below 3\% the intensity of the continuum. Four different heliocentric angles are overplotted. The right slope of the histograms shows no dependence on the heliocentric angle, while in the left branch histograms from profiles at less than 30 degrees $(\mu>0.86)$ show lower values.

regions. Simulation of these scenarios has successfully reproduced these single-lobed profiles with strong area asymmetries. We should therefore distinguish among two different magnetic scenarios: the turbulent field we are interested in studying and the single-lobed Stokes V profiles appearing at the boundaries of concentrations with strong magnetic field. It is essential to be able to distinguish between these two classes of profiles by looking at the statistics of the asymmetries alone. Consequently, in Fig. 4, we try to ascertain the contribution of single lobe profiles to our histograms. Since it is difficult to see the true weight of profiles with a given asymmetry in a histogram with a logarithmic scaling, we computed the 95 percentile of the area asymmetry, that is, the value of the asymmetry such that $95 \%$ of the profiles with the same amplitude have an area asymmetry smaller than, or equal to, that value. We see in the left column of Fig. 4 that the 95 percentile area asymmetry is almost constant for all these fields with amplitudes below 3\%. That is, once we enter into the class of weak fields, profiles with extreme asymmetries beyond 0.7 contribute to the histograms with less than $5 \%$ of the cases, and that is independent of amplitude or heliocentric angle. We consider that given the measurement conditions and the rough proxy that the 95 percentile is, the constant value of 0.7 is to be identified as the microturbulent asymmetry limit. We conclude therefore that $95 \%$ of the profiles with weak amplitudes have asymmetries that can be explained as radiative transfer of polarized light through a turbulent magnetic field. The constancy of this value for any amplitude and any heliocentric angle is in our view a strong statement in favor of this identification. What other explanation as simple as the one we put forward can be offered to explain that asymmetry values fall predominantly in the range (0-0.7) independent of both signal amplitude (i.e. longitudinal flux density) and heliocentric angle?

Summarizing, in this section we have successfully identified three different magnetic regimes in the data of area asymmetries: first, a strong, coherent, organized, mostly vertical and weakly asymmetric field, second, the anomalous single-lobed Stokes V profiles with strong asymmetries contributing to less than $5 \%$ of the cases, which are located in the boundaries of strongmagnetic field concentrations, and finally a turbulent field that is quasi-isotropic and has asymmetries in the range expected from stochastic radiative transfer calculations. With this classification we reach the first and primary result of the present work, which is that also through area asymmetries we identify the presence of a turbulent, quasi-isotropic magnetic field component in the quiet Sun. Asymmetries are sensitive to the variations of the magnetic and velocity field along the line of sight. This is complementary to previous studies that concentrated on statistics of amplitudes and amplitude ratios that were sensitive to magnetic fields placed side by side over the photosphere. Despite this complementarity, we arrive at the same conclusion of the presence of a turbulent and isotropic field in the quiet Sun.

\section{Scales of the turbulent magnetic field in the quiet Sun}

We now focus on the regime of turbulent fields identified in the statistical data of area asymmetries. These fields are mostly isotropic, as is to be expected from turbulence associated to the photospheric plasma, and they have low amplitudes. They should not be interpreted as the direct, which is best described by a probability distribution function (Trujillo Bueno et al. 2004; Domínguez Cerdeña et al. 2006; López Ariste et al. 2007; Sánchez Almeida 2007; Sampoorna et al. 2008; Stenflo 2010). A good choice for that distribution function is a Maxwellian for the modulus of the vector (the field strength) (Domínguez Cerdeña et al. 2006; López Ariste et al. 2007; Sánchez Almeida 2007), which is fully isotropic for its inclination and azimuth in whatever reference system of choice. The average value of this probability distribution function for a vector field is the null vector. But it would be a mistake to interpret from that zero average that no polarization signals would be observed if this were the topology of the field in the quiet Sun. The average of the distribution is only attained at the limit of infinite realizations. Only if the scale of variation of the magnetic field, along the line of sight or across our resolution element, were zero the average would be realized and observed. But in reality that scale of variation is nonzero, and both along the formation region of the observed spectral line and across the spatial resolution element of our observation, a finite number of magnetic field realizations is found. Their integration will fluctuate around the average zero value, but will not be zero. Those fluctuations are what is to be expected to become the observed of longitudinal flux density in a turbulent magnetic field scenario. The variance of those fluctuations will diminish with the square root of the number of realizations. Therefore one should expect in the approximation of magnetic realizations sitting side by side across the spatial resolution element that as the spatial resolution increases, the average longitudinal flux increases not with the square of the size of the resolution element (as could be expected if unresolved magnetic structures were present), but linearly with that size. This is what is indeed observed in the quiet Sun, where observations of the average flux density in the quiet Sun have shown no particular difference between instruments with $1^{\prime \prime}, 0.6^{\prime \prime}$ or $0.3^{\prime \prime}$ resolutions (Martínez González et al. 2010). This is one of the strongest arguments in favor of a turbulent magnetic field in the quiet Sun.

In terms of area asymmetries we are concerned about the number of realizations of the magnetic probability distribution function along the formation region of the observed line. Contrary to the case of the spatial resolution, it is not evident to change the size of the sampling region. The formation region of a spectral line is what it is and cannot be changed and, in the photosphere, most spectral lines of interest have formation regions that are too similar in size and position to be of any interest in the present terms. However, it is of great advantage that 
A. López Ariste and A. Sainz Dalda: Scales of the magnetic fields in the quiet Sun
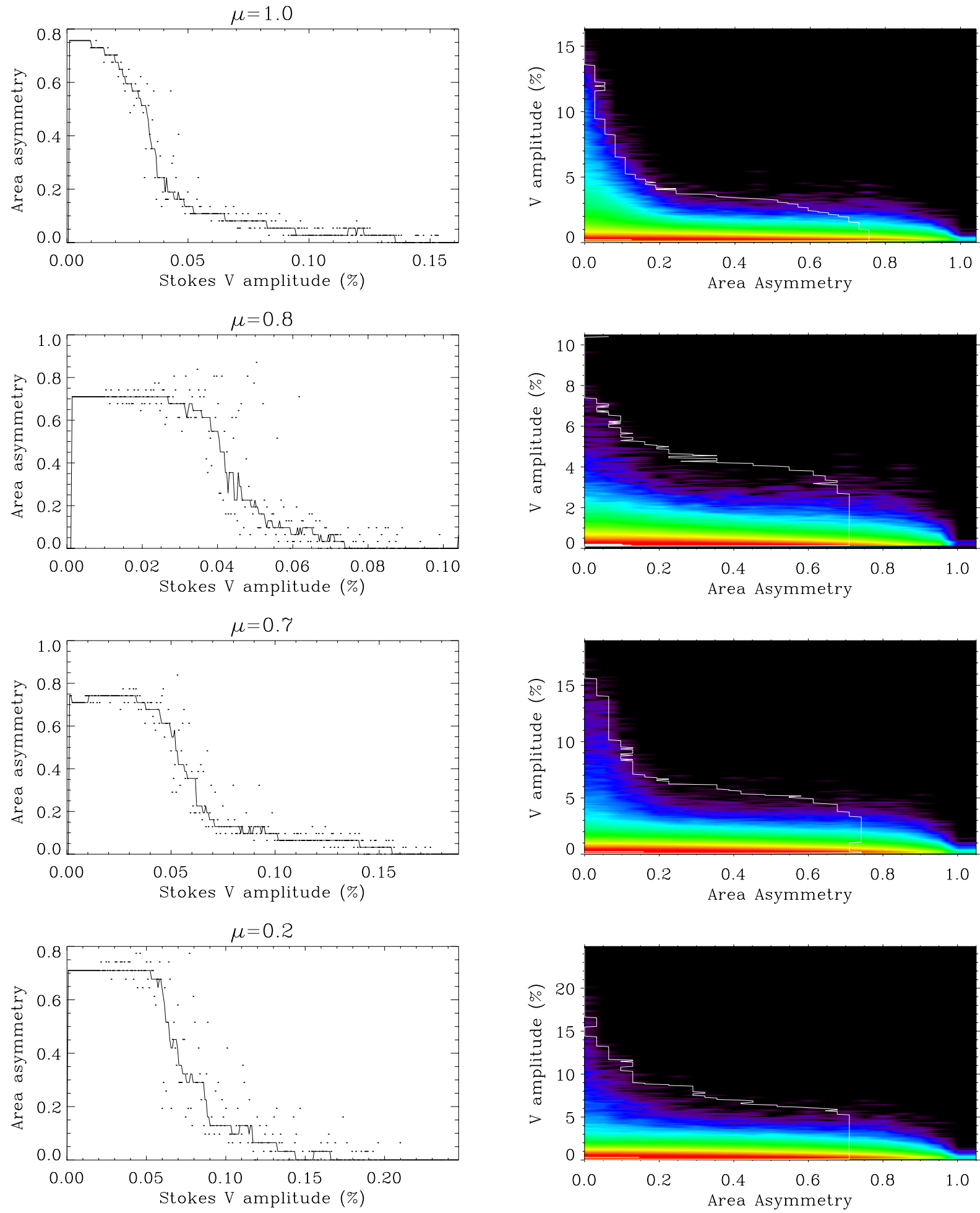

Fig. 4. 95\% percentile value of the unsigned area asymmetry of Stokes $V$ for different heliocentric angles and as a function of the amplitude of the signal. In the left column we present the actual measurements with a smoothed median line overplotted. In the right column this median line has been drawn over the 2D unsigned histograms of Fig. 1.

the size of those formation regions is quite small, a few hundred kilometers at most, and the response functions over those regions are not flat but present a shape that has led many authors to speak of height of formation rather than region of formation despite warnings about this oversimplification (Sanchez Almeida et al. 1996). Therefore, when observing area asymmetries we are sampling those small regions of formation. We are directly sampling scales of less than $100 \mathrm{~km}$. These scales are difficult to access for any diffraction-limited imaging instrument. Therefore area asymmetries present a clear advantage in terms of sensing the scales of variation of turbulent magnetic fields.

To correctly interpret the actual values of area asymmetries in terms of scales of variation of the magnetic field, we require a theory for radiative transfer of polarized light through 


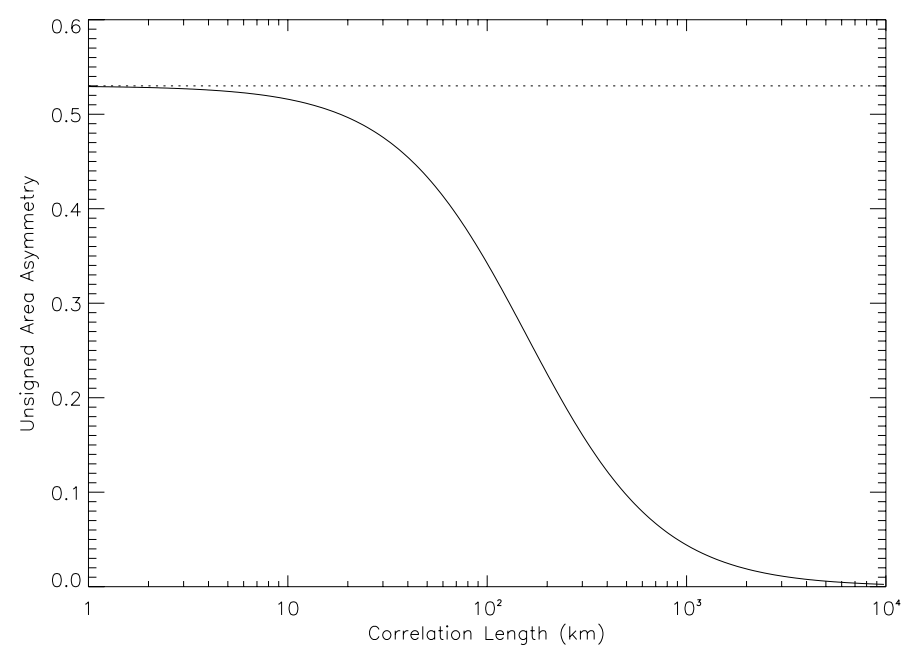

Fig. 5. Absolute value area asymmetry as a function of the correlation length obtained after resolving the radiative transfer equation of polarized light through stochastic atmospheres. The dashed line at $\sim 53 \%$ (or 0.53) marks the microturbulent limit for asymmetry parameters calculated under the MISMA approximation (data from Carroll \& Kopf 2007, right panel of Fig. 1).

stochastic atmospheres. Such models can be found in the works of Auvergne et al. (1973); Frisch \& Frisch (1976); Frisch et al. (2005, 2006) and Carroll \& Staude (2005), to give a few solarrelated examples. Here we used the result of Carroll \& Kopf (2007) since they explicitly computed area asymmetries as a function of the correlation length of the parameters in their stochastic atmospheres. The correlation length, the key concept for the goal of this paper, describes the distance between two points along the light path for which the probability of the magnetic field being the same is small ${ }^{1}$, assuming a Markovian model in which this probability falls with increasing distance. This correlation length describes "the mean length scale of the structures", to quote Carroll \& Kopf (2007). Roughly in every correlation length along the light path there is a change in the value of the atmospheric parameters, and gradients consequently appear producing asymmetries in the profiles. If the correlation length is longer than the formation region of the line, there will be no change in parameters, the magnetic and velocity fields will be constant and no asymmetries must be expected. With a shorter correlation length than the formation region, more gradients appear and accordingly asymmetry grows. However, if the correlation length shrinks beyond the mean free path of the photon in the atmosphere, there are no atom-photon interactions to take those small-scale gradients into account and the asymmetries saturate to a microturbulent limit. This is the expected dependence of asymmetries with correlation length; and this is what Carroll \& Kopf (2007) found in their calculations, shown in their Fig. 1 (right side), which we reproduce here in Fig. 5 for the sake of completeness. The details on the computation of the figure are given by the authors of the referred work. The figure refers to the same Fe I line as used in the observations of the present work. Using the computed relation shown in that figure but reversing the argument, the observed asymmetries of those profiles (identified as belonging to the turbulent regime in the previous section) can be translated into correlation lengths. Since all asymmetries from 0 to the micro turbulent limit of 0.53 are observed, we can already conclude that in the data considered

\footnotetext{
${ }_{1}$ In the particular model adopted by Carroll \& Kopf (2007), this probability is $1 / e$.
}
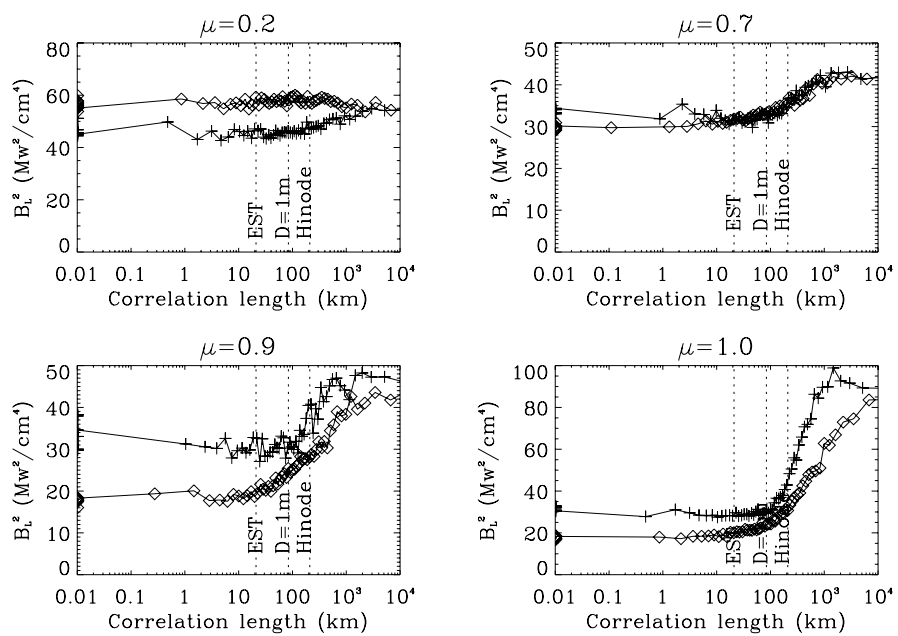

Fig. 6. Estimates of the magnetic energy spectrum of the turbulent magnetic field of the quiet Sun observed through the area asymmetries of Stokes V. For each heliocentric angle, the two curves correspond to positive (crosses) and negative (diamonds) asymmetries. The diffraction limits of three characteristic solar instruments are given for reference.

for this work there are profiles formed in regions with variations of the magnetic field at scales of $10 \mathrm{~km}$. Below that length the asymmetries quickly and asymptotically approach the microturbulent limit and we loose our sensitivity. It is important, before proceeding any further, to stress that point: the observation of asymmetries in the Stokes $\mathrm{V}$ profiles allows us to identify tiny scales of variation for the magnetic fields that produce those signals. Many of the strong signals identified in the previous section with the structured and mostly vertical fields presented asymmetries around zero. That is, those structures had correlation lengths or were coherent over scales of hundreds of $\mathrm{km}$ up to $1000 \mathrm{~km}$. This is what we should expect from structured fields and it is justified that the best instruments in terms of spatial resolution start resolving them more and more frequently.

On the other hand, for the fields that we assigned to the turbulent regime, the range of variation of observed asymmetries fills the allowed range of variation all the way up to the microturbulent limit. Thus we have identified profiles that arise from regions with magnetic fields varying at scales of less than $100 \mathrm{~km}$ and eventually down to $10 \mathrm{~km}$. These are actual observations, not simulations or estimates. To put those scales in another context, they correspond to the diffraction limit of an instrument with $7 \mathrm{~m}$ of diameter of entrance pupil. More interesting, it is comparable to, or smaller than, the mean free path of the photon (Mihalas 1978; Sanchez Almeida et al. 1996). We emphasize these comparisons because they show the finesse of the diagnostic that is accessible through the asymmetries of the Stokes V profile.

After translating the asymmetry of each profile belonging to the turbulent regime to correlation lengths, we noticed that we also have a measurement of the longitudinal flux density. It is tempting to try and convert it into magnetic energy which would allow us to produce the all-important magnetic energy spectrum of the turbulent magnetic field (Nakagawa \& Priest 1973; Knobloch \& Rosner 1981).

There is a caveat attached to this, however. It is a main result of this work to have identified the three different regimes as seen from the point of view of area asymmetries in the Stokes V profiles. For the turbulent regime, the actual asymmetry obviously depends on the correlation length of the parameters, including the magnetic and velocity fields, of the stochastic atmosphere used in the model. The details of the stochastic radiative transfer 
may be a matter of discussion and study, but the general relation of asymmetries with correlation length is not in doubt. Our claim to have measured magnetic fields varying at scales below $100 \mathrm{~km}$ is also a robust result of this work. To proceed to a magnetic energy spectrum requires a series of assumptions, approximations and simplifications, which may appear as too strong. We nevertheless proceed because 1) those simplifications and approximations are, in our opinion, still justified as fairly educated guesses, and 2) because the final result, even if crude, still illustrates how powerful a tool the analysis and study of area asymmetries can be.

With those cautions, we assume that the measured longitudinal flux density is a fair proxy of the average field strength of the distribution of fields present along the line of sight. Because of the projection along the line of sight we can assume that it is indeed a lower bound to that field strength. We furthermore assume that the probability distribution function underlying the observed distribution is Maxwellian for the field strength. This distribution is fully determined by the position of its unique maximum. Our measurement of the longitudinal flux density is seen as a lower boundary proxy to that maximum. The magnetic energy of the Maxwellian is computed as

$E=\frac{\int B^{2} p(B) \mathrm{d} B}{\int p(B) \mathrm{d} B}$,

where $p(B)$ is the Maxwellian probability distribution function. The integrand looks in shape very much like the original Maxwellian, but its maximum is shifted toward higher values and the high-field wing is emphasized. Because of these similarities we can claim that the integrand is fully determined by the new maximum of which the maximum of the original Maxwellian is a lower boundary. Our measurement of the longitudinal flux density therefore provides a lower limit to the magnetic energy in the distribution of fields along the line of sight in our turbulent atmosphere. In Fig. 6 we plot at four different heliocentric angles this magnetic energy as a function of the correlation length. They are in a sense crude approximations to the magnetic energy spectrum with sensitivities down to scales of $10 \mathrm{~km}$.

Owing to the approximations and simplifications made it is difficult to extract much information from those energy spectra. Clearly, where the strong and structured fields are seen, near disk center, they dominate the energy spectrum and create a bump at the larger scales and a slope toward smaller scales that can be compared to previous results (Nakagawa \& Priest 1973). But that appears to be a valid description only for the more organized and mostly vertical fields. For the fields that we included in the turbulent regime, the spectrum appears to be decreasing over all the scales at which we are sensitive with a tendency to flatten out toward the $10 \mathrm{~km}$ limit and certainly below that limit, where we are not sensitive.

\section{Conclusion}

We studied the properties of the turbulent magnetic field that we assumed to pervade most of the quiet Sun. It is not the purpose of this work to demonstrate the existence of this turbulent component. Many observational and theoretical arguments are accumulating to prove its existence, one of which is the way in which we are detecting it through Zeeman polarimetry. We added new observational arguments confirming its existence but, beyond that, we studied it through the asymmetries in the Stokes V. We have collected and analyzed the asymmetries of the Stokes V profile of the Fe I line at $6302.5 \AA$ observed with Hinode-SOT/SP at several heliocentric angles. The histograms of those asymmetries for different signal amplitudes (or longitudinal flux densities) reveal three different magnetic regimes. We notice that although in our results we talk about three regimes as separated, this is only a classification to facilitate the understanding of their existence. Needless to say, these regimes are simultaneously taking place in the quiet Sun and they are not uniquely separated by the area asymmetry value but also by their flux density or magnetic energy spectrum. The first one is made of strong signals with weak area asymmetries that are mostly distributed around zero. They are mostly vertical, as can be concluded from the field's importance at disk center and its waning as we approach the limb. The weak asymmetries, when interpreted in terms of magnetic and velocity field variations along the line of sight, are a signature of big correlation lengths, that is, these structures present coherent fields all throughout them, which justifies referring to them as magnetic structures.

The second magnetic regime detected is characterized by its weak longitudinal flux density (below $30 \mathrm{MW} / \mathrm{cm}^{2}$ at HinodeSOT/SP spatial resolutions) and unsigned area asymmetries that span the full range from 0 through the observed microturbulent limit of 0.7 . This is slightly higher than the theoretically predicted value of 0.53 . We identified them as the turbulent field that pervades the quiet Sun. But before focusing on this, we first turn to the third magnetic regime detected, also made of weak signals, but with strong asymmetries beyond the microturbulent limit. Distinguishing between these two regimes is a delicate matter. It is done through two independent observations. The first is that these single-lobed Stokes V profiles with area asymmetries beyond 0.6 or 0.7 are to be found mainly in the boundaries of strong magnetic field concentrations. This observation has prompted us to interpret them as profiles arising from lines of sight that cross from a strong magnetic structure to the turbulent quiet Sun. These jumps in the magnetic and thermodynamic parameters of the atmosphere along the line of sight can produce anomalous profiles like this. The second observation to tell them apart from the turbulent regime is the 95 percentile of the asymmetry histogram. This statistical measure is in the data independent of the amplitude signal or heliocentric angle and roughly equal to the microturbulent limit. The independence of the amplitude signal is the hardest test that makes us confident that the two regimes can be also directly separated in the histograms.

As a first result of this work we identified the magnetic turbulent fields in the data of area asymmetries in the Stokes V profiles. We furthermore realized that the histograms of the asymmetries of these profiles are almost independent of the heliocentric angle, which is another observational result supporting the isotropy of these fields. Next we used the results of radiative transfer of polarized light through stochastic atmospheres, particularly the work of Carroll \& Kopf (2007) to translate those asymmetries into correlation lengths for the atmospheric parameters, specifically the magnetic and velocity fields. We stressed the sensitivity to small scales that area asymmetries provide. The formation region of the observed spectral lines spans a few hundred kilometers and is mostly concentrated in a few tens of kilometers. This is much better than the spatial resolutions achievable through direct imaging. We emphasized that we measured scales of variation and not physical structures. Those scales of variation are the best description of a turbulent field, without identifiable structures over the range of scales corresponding to the inertial regime of the turbulence. The smallest of these scales is the scale of dissipation, which has been estimated to be as 
small as $100 \mathrm{~m}$ (Graham et al. 2009). Our measurements appear to identify magnetic scales that are still short of this dissipation scale by a factor 100 .

With a powerful tool like this we determined the range of scales of variation of the turbulent magnetic field and saw that examples of all scales are found: from the hundreds of kilometers of magnetic concentrations that other instruments start to resolve through direct imaging through the tiniest scales of $10 \mathrm{~km}$ at which area asymmetries loose their sensitivity. In an effort to exploit this information on scales, we attempted to measure the magnetic energy through the proxy of the longitudinal flux densities. There are many approximations and simplifications, but beyond the confidence on the final result we stress again the potential of the tool if only better determinations of the magnetic energy can be used.

Acknowledgements. Hinode is a Japanese mission developed by ISAS/JAXA, with NAOJ as domestic partner and NASA and STFC(UK) as international partners. It is operated in cooperation with ESA and NSC (Norway). The Hinode project at Stanford and Lockheed is supported by NASA contract NNM07AA01C (MSFC)

\section{References}

Asensio Ramos, A. 2009, ApJ, 701, 1032

Auvergne, M., Frisch, H., Frisch, U., Froeschle, C., \& Pouquet, A. 1973, A\&A, 29, 93

Carroll, T. A., \& Kopf, M. 2007, A\&A, 468, 323

Carroll, T. A., \& Staude, J. 2005, Astron. Nachr., 326, 296

Domínguez Cerdeña, I., Sánchez Almeida, J., \& Kneer, F. 2006, ApJ, 636, 496

Frisch, H., \& Frisch, U. 1976, MNRAS, 175, 157

Frisch, H., Sampoorna, M., \& Nagendra, K. N. 2005, A\&A, 442, 11

Frisch, H., Sampoorna, M., \& Nagendra, K. N. 2006, A\&A, 453, 1095
Graham, J. P., Danilovic, S., \& Schüssler, M. 2009, ApJ, 693, 1728

Knobloch, E., \& Rosner, R. 1981, ApJ, 247, 300

Kosugi, T., Matsuzaki, K., Sakao, T., et al. 2007, Sol. Phys., 243, 3

Lites, B. W. 2002, ApJ, 573, 431

López Ariste, A. 2002, ApJ, 564, 379

López Ariste, A., Malherbe, J. M., Manso Sainz, R., et al. 2007, in SF2A2007: Proceedings of the Annual meeting of the French Society of A\&A, ed. J. Bouvier, A. Chalabaev, \& C. Charbonnel, 592

Martínez González, M. J., Asensio Ramos, A., Carroll, T. A., et al. 2008a, A\&A, 486, 637

Martínez González, M. J., Asensio Ramos, A., López Ariste, A., \& Manso Sainz, R. 2008b, A\&A, 479, 229

Martínez González, M. J., Manso Sainz, R., Asensio Ramos, A., López Ariste, A., \& Bianda, M. 2010, ApJ, 711, L57

Martínez Pillet, V., Lites, B. W., \& Skumanich, A. 1997, ApJ, 474, 810

Mihalas, D. 1978, Stellar atmospheres, 2nd edn. (San Francisco: W. H. Freeman and Co.)

Nakagawa, Y., \& Priest, E. R. 1973, ApJ, 179, 949

Nordlund, A., Brandenburg, A., Jennings, R. L., et al. 1992, ApJ, 392, 647

Parker, E. N. 1982, ApJ, 256, 292

Petrovay, K. 2001, Space Sci. Rev., 95, 9

Sainz Dalda, A., Martínez-Sykora, J., Bellot Rubio, L., \& Title, A. 2012, ApJ, 748, 38

Sampoorna, M., Nagendra, K. N., Frisch, H., \& Stenflo, J. O. 2008, A\&A, 485, 275

Sánchez Almeida, J. 2007, ApJ, 657, 1150

Sánchez Almeida, J., \& Lites, B. W. 1992, ApJ, 398, 359

Sanchez Almeida, J., Ruiz Cobo, B., \& del Toro Iniesta, J. C. 1996, A\&A, 314, 295

Solanki, S. K., \& Pahlke, K. D. 1988, A\&A, 201, 143

Solanki, S. K., \& Stenflo, J. O. 1984, A\&A, 140, 185

Stenflo, J. O. 1973, Sol. Phys., 32, 41

Stenflo, J. O. 2010, A\&A, 517, A37

Trujillo Bueno, J., Shchukina, N., \& Asensio Ramos, A. 2004, Nature, 430, 326

Tsuneta, S., Ichimoto, K., Katsukawa, Y., et al. 2008, Sol. Phys., 249, 167

Viticchié, B., Sánchez Almeida, J., Del Moro, D., \& Berrilli, F. 2011, A\&A, 526, A60 